CERN-PPE/94-19

INFN-AE/94/04

1 February 1994

\title{
Construction and test of calorimeter modules for the CHORUS experiment
}

\author{
S. Buontempo ${ }^{\text {b) }}$, A. Capone ${ }^{\mathrm{c})}$, A. G. Cocco $^{\text {b) }}$, D. De Pedis ${ }^{\mathrm{c}}$, E. Di Capua ${ }^{\mathrm{a})}$, \\ U. Dore $^{\text {c) }}$, A. Ereditato ${ }^{\text {b) }}{ }^{*}$, M. Ferroni ${ }^{\text {a) }}$, G. Fiorillo ${ }^{\text {b) }}$, P. F. Loverre ${ }^{\text {c) }}$, \\ C. Luppi ${ }^{\mathrm{a})}$, D. Macina ${ }^{\mathrm{c})}$, F. Marchetti-Stasi ${ }^{\mathrm{b})}$, M. A. Mazzoni ${ }^{\mathrm{c}}$, \\ P. Migliozzi ${ }^{\text {b) }}$, V. Palladino ${ }^{\text {b) }}$, G. Piredda $^{\text {c) }}$, F. Riccardi ${ }^{\text {b) }}$, S. Ricciardi $^{\text {a) }}$, \\ P. Righini ${ }^{\text {c) }}$, B. Saitta ${ }^{\text {a) }}$, R. Santacesaria ${ }^{\text {() }}$, P. Strolin ${ }^{\text {b) }}$ and P. Zucchellia) \\ a) Università and Sezione INFN, Ferrara, Italy \\ b) Università "Federico II" and Sezione INFN, Napoli, Italy \\ c) Università "La Sapienza" and Sezione INFN, Roma, Italy
}

\begin{abstract}
The construction of modules and the assembly of the calorimeter for CHORUS, an experiment that searches for $\nu_{\mu} \longleftrightarrow \nu_{\tau}$ oscillation, have been completed. Within the experiment, the calorimeter is required to measure the energy of hadronic showers produced in neutrino interactions with a resolution of $\sim \frac{30 \%}{\sqrt{E(G e V)}}$. To achieve this performance, the technique, developped in recent years, of embedding scintillating fibers of $1 \mathrm{~mm}$ diameter into a lead matrix has been adopted for the most upstream part of the calorimeter. A more conventional system, of alternating layers of lead and scintillator strips, was used for the rest. Details of module construction as well as results obtained when modules were exposed to electron and muon beams are presented.
\end{abstract}

\footnotetext{
*) Currently at CERN, Geneva, Switzerland.
} 
It has been found $[1,2]$ that fibers of $1 \mathrm{~mm}$ diameter and a volume ratio of 1, with lead as passive and scintillator as active materials assure both compensation d good sampling, resulting in an energy resolution of $\sim 13 \% / \sqrt{E(G e V)}$ and of $\sim$ $\% / \sqrt{E(G e V)}$ for electromagnetic and hadronic showers respectively.

The calorimeter described in this paper is the first large scale application of such rechnique. However, this calorimeter differs from those studied so far for the fibers are aced in a direction transverse to the beam direction, a configuration seldom used up now. This is required by the function that the calorimeter has to perform within the periment and constitutes a novelty of the device.

This paper is organized as follows. The conceptual design of the complete lorimeter is presented in Section 2. Details of the construction of the three types modules which are used are given in Section 3. Section 4 contains a description of e experimental setup where some of these modules were exposed to charged particle ams. In Section 5 the first results on the performance of the individual modules are scussed and conclusions are drawn in Section 6.

\section{Design of the calorimeter}

The CHORUS experiment [3] searches for $\nu_{\mu} \longleftrightarrow \nu_{\tau}$ oscillations in the CERN SPS de band neutrino beam.

Possible charged current $\nu_{\tau}$ interactions, with the subsequent decay of the $\tau$ ton into a muon or pions, are searched for in a target of about $0.8 t$ of nuclear emulons, which is followed by the electronic components of the detector (scintillating fiber ackers, an air-core magnet, the calorimeter described in this paper and a muon specometer). $\tau$ decay candidates have to be isolated from a large background of ordinary interactions and it is essential to reduce as much as possible, by means of electronic tectors, the number of events to scan in the nuclear emulsions. This reduction can be hieved by means of a selection on kinematic variables, since the presence of a neutrino the decay results in an unbalance of the visible transverse momentum. Such a selecon is more effective if the hadronic shower produced in the interaction is measured th high energy and angular resolution.

It is also important to have the capability to track through-going muons and to atch their trajectories with those determined in the other detectors of the apparatus, requirement that has not played an important role in the applications, considered so , of calorimeters of this type at colliders. Therefore the calorimeter must be a tracking vice, with the fibers placed in a direction perpendicular to that of the incident particles d longitudinal segmentation is also necessary to allow for the insertion of limited ceamer tube chambers. Furthermore, the size of the neutrino beam requires modules considerable length. This makes the problems generated by the attenuation of the ht - that has to travel through considerable distances to reach the photomultipliers tentially more serious than in other calorimeters based upon the same principle, but th a longitudinal orientation of the fibers.

The calorimeter designed for the CHORUS experiment satisfies the requirements 
itted in the interactions with good accuracy the width of the modules was chosen to much smaller than the size of the average hadron shower.

The calorimeter consists of three sectors with decreasing granularity, called EM, ID1 and HAD2. The first sector will measure the electromagnetic component of the dronic shower in the neutrino induced events, while the other two sectors will comete the measurement of the hadronic component. The total depth is about 5.2 intertion lengths, sufficient to contain $99 \%$ of the shower produced by a pion of $5 \mathrm{GeV} / \mathrm{c}$ omentum. It should be observed that $\sim 90 \%$ of the hadrons produced in the neutrino teractions have momentum less than $5 \mathrm{GeV}$.

The first two sectors of the calorimeter are made of scintillating fibers and lead, e third sector is made as a sandwich of lead and scintillating strips. The structure the calorimeter allows light collection through photomultipliers on both sides of the odule and this is exploited to reduce effects of light attenuation.

Between the horizontal and the vertical planes of calorimeter modules, limited ceamer tube planes are inserted to allow tracking of penetrating charged particles and e arranged in pairs (to have two coordinates). The streamer tubes were previously used c the CHARM II experiment and their characteristics and performances are described [5]. In CHORUS, however, only the digital signals from the wires are recorded.

The main features of the calorimeter are summarized in Table 1 and its isometric ew is shown in Fig.1. It is mounted on a support standing on rails, so that it can be oved off the detector axis to perform dedicated energy calibration with electron and on beams.

\section{Module construction}

A careful check on the quality of the scintillating materials was performed. All e 900 scintillator strips and a sample, corresponding to $4 \%$ of the fibers, were tested, verify that individual attenuation length and light output were adequate for our Irposes. For the fiber tests, a special device was built at INFN Naples [6], while more nventional means ( $\beta$-source and multi channel analyser) were used for the strips.

\section{Electromagnetic modules}

The electromagnetic sector is composed of two horizontal and two vertical planes 31 modules each. These are built by piling up extruded layers of grooved lead and astic scintillating fibers positioned in the grooves. A module consists of a pile of 21 yers, $2620 \mathrm{~mm}$ long and $82.4 \mathrm{~mm}$ wide, and 740 fibers of $1 \mathrm{~mm}$ diameter and $3050 \mathrm{~mm}$ 


\begin{tabular}{|c|c|c|c|c|}
\hline & EM & HAD1 & HAD2 & Total \\
\hline Module Dimension $(\mathrm{mm})$ & $40 \times 80 \times 2620$ & $80 \times 80 \times 3350$ & $100 \times 200 \times 3690$ & - \\
\hline Number of Planes & 4 & 5 & 5 & 14 \\
\hline Depth: $X_{\circ}\left(\lambda_{i n t}\right)$ & $21.5(0.78)$ & $55.2(2.0)$ & $67.1(2.44)$ & $143.8(5.22)$ \\
\hline Number of Modules & 124 & 200 & 90 & 414 \\
\hline M Type & $\begin{array}{c}\text { Hamamatsu } \\
\text { R1355/SM }\end{array}$ & $\begin{array}{c}\text { Thorn - EMI } \\
9839 \mathrm{~A}\end{array}$ & $\begin{array}{c}\text { Thorn - EMI } \\
\text { 9839A }\end{array}$ & - \\
\hline M per Module & 4 & 2 & 4 & - \\
\hline Total Number of PM & 496 & 400 & 360 & 1256 \\
\hline Fibers/Strips Type & $\begin{array}{l}\text { Kuraray } \\
\text { SCS-F81 }\end{array}$ & $\begin{array}{c}\text { Bicron } \\
\text { BCF-12 }\end{array}$ & $\begin{array}{c}\text { Bicron } \\
\text { BC-408 (strips) }\end{array}$ & - \\
\hline Number of fibers/strips & 93000 & 310800 & 900 & 404700 \\
\hline Total Length $(\mathrm{km})$ & 283 & 1165 & 3 & 1451 \\
\hline b Weight (tons) & 9.7 & 42 & 60 & 111.7 \\
\hline cintillator Weight (tons) & 0.22 & 0.92 & 1.32 & 2.46 \\
\hline
\end{tabular}

Table 1: Characteristics of the calorimeter. 
neter is $1.1 \mathrm{~mm}$ and the sheet thickness $1.9 \mathrm{~mm}$. The layer material is $99 \%$ lead with 6 antimony content, which improves its mechanical properties. An overall thickness suniformity of less than $2 \%$ was achieved through the extrusion procedure.

The fibers were manufactured by Kuraray and are of the type SCSF81. They nsist of a polystyrene core surrounded by a $30 \mu \mathrm{m}$ thick acrylic cladding, and emit the blue, with a maximum around $420 \mathrm{~nm}$. A reduction in the light attenuation is tained by painting the surface of the last 5 centimeters of the fibers (on each side) by rylic black paint. This has the effect of reducing the light from the cladding, which has smaller attenuation length. About 93000 fibers were used to build the electromagnetic odules, for a total length of $280 \mathrm{~km}$.

The construction is performed by alternating on a L-shaped steel half-box $(0.8 \mathrm{~mm}$ ick) lead and fiber layers. After the last (lead) layer the second half-box is positioned. double-sided special adhesive paper is glued between the lateral internal face of the cond half-box and the edge of the lead sheet pile. The box is finally closed by soldering e two halves, kept together by applying an appropriate pressure. The adhesive paper needed to prevent sliding of the layers in the vertical modules; in addition, a ridge echanically blocks the layers.

Once the box is closed, fibers are grouped at both ends into two sets - so as to $\mathrm{cm}$ the two read-out cells - and the faces of the bundles are finally milled and polished. ne cross-section of the fiber bundles is hexagonal $(22.2 \mathrm{~mm}$ apex to apex) and they e coupled to a plexiglas light guide, also with hexagonal cross-section $(24 \mathrm{~mm}$ apex to ex). The hexagonal shape and the length of the light guide have been chosen to reduce suniformities in the mixing of the light coming out of the individual fibers [1]. The ht guides are then coupled to 1" photomultipliers, type R1355/SM from Hamamatsu, th a special green extended photocathode, of $24 \mathrm{~mm}$ useful diameter. The window of e photomultiplier is covered by a yellow filter Kodak Wratten n.3, which absorbs light th wavelength less than $450 \mathrm{~nm}$ and therefore selects the spectral component which s larger attenuation length. Two aluminum boxes (one at each side) house pairs of ht guides, photomultipliers, voltage dividers, and mumetals. The boxes are light tight d are closed by PVC plates holding the high voltage and signal connectors.

The EM modules were constructed in the laboratories of INFN Naples.

\section{HAD1 modules}

Each of the five planes of the HAD1 part of the calorimeter is formed by 40 odules, each made of 43 extruded layers of lead identical in height and groove size to ose used for the EM sector, but with a length of $3350 \mathrm{~mm}$. (see Fig. 4). The grooves 
Fibers are collected at both ends in an hexagonal bundle whose polished end is upled, via a light pipe, to a 2" photomultiplier (EMI 9839 A), previously used in the IARM II experiment. The empty space of the boxes around the fiber bundles is filled th an epoxidic resin (Araldit D) with microballons of a phenolic resin as an additive, avoid sliding of the lead sheets in vertical modules. This mixture contains also a nall percentage of carbon black to reduce light leaks and the contribution of light from e cladding, playing therefore the same role as that of the black paint in the EM type odules.

The modules were assembled by Pol.Hi.Tech. at Carsoli (Italy), using a semithomatic device developed by INFN Rome. The steps followed at assembly stage are nilar to those employed for the construction of electromagnetic modules; the steel $\mathrm{x}$, in this case, is compressed with a total force of $9 \times 10^{4} \mathrm{~N}$. The fibers are collected gether and are extracted from the terminal parts of the boxes. At this stage the filling ixture, whose hardening time is about 24 hours, is poured in. The faces of the bundles e then milled and polished and a first test of optical quality of the whole assembly is ade. For mechanical reasons, the lead sheets of EM and HAD1 modules have a different ientation relative to the beam direction, as illustrated in Fig. 2 and 4.

\section{HAD2 modules}

The HAD2 sector is made of five planes (three vertical and two horizontal) of 18 odules each, for a total of 90 modules and a mass of about $60 t$.

Each module is constructed by superposing five alternate layers of one lead bar ade of $99 \%$ lead and $1 \%$ antimony $(3690 \times 200 \times 16 \mathrm{~mm})$ and two adjacent scintillator cips (BICRON, BC-408) $3714 \times 100 \times 4 \mathrm{~mm}$ packed in a $0.5 \mathrm{~mm}$ thick stainless steel box en at both ends (see Fig. 5), made of two halves held together by rivets.

Within one module, each of the two groups of five scintillator strips is coupled to photomultipliers - of the same type used for HAD1 - at both ends via plexiglas light ides; therefore a single module is seen by a total of four photomultiplier tubes, and us contains two read-out cells. Details of the optical coupling of the scintillator to the otomultiplier are shown in Fig. 6. Five trapezoidal, laser-cut, plexiglas plates, $6 \mathrm{~mm}$ ick, $200 \mathrm{~mm}$ long and $100 \mathrm{~mm}$ and $40 \mathrm{~mm}$ bases, are glued to the scintillator strips prior their insertion in the calorimeter modules, using an optical cement (BC-600) with an dex of refraction close to that of the scintillator. A groove of $4 \mathrm{~mm}$ width and $2 \mathrm{~mm}$ pth forming an angle with the vertical plane - varying according to the position of the ht guide in the vertical stack (see Fig. 6) - was machined at the wider end to ensure good contact with the scintillator.

A special device was constructed to allow the simultaneous glueing of two sets of 
ht tight wrapping about $0.5 \mathrm{~mm}$ thick, reflective on the inside; the plexiglas guides re made light tight by using a foil of black plastic material with an aluminised inner ating, provided by Prodicon.

The attenuation length and light yield of each scintillator strip were measured, fore glueing, using a radioactive source; it was found that the distributions had a read about their mean value of $13 \%$ and $5 \%$, respectively.

Because of their weight, the HAD2 modules were assembled at CERN, close the e of the CHORUS apparatus.

\section{Test beam setup}

While a check on the quality of nearly all the modules was performed using cosmic ys, some were exposed to a charged particle beam to gather detailed information on eir expected performance and resolution.

A testing station was prepared in the X7B area of the X7 test beam at CERN, d data were taken in November 1992 and May 1993 with electrons, positrons and uons, the test with pions being meaningful only with the assembled calorimeter, which ovides an adequate number of interaction lengths.

The setup is shown in Fig. 7. Coincidences between the scintillation counters $\cdot T_{2} \cdot \bar{H}$ (and when required $T_{3} \cdot T_{4}$ ) constituted the main trigger. The data acquisition as based on a FIC 8232 system (running SPIDER under OS9) and communicating with AMAC through a VIC bus [7]. Real time monitoring and analysis were possible on a DEC ax Station connected to the acquisition setup through ethernet. The analog signal from e photomultipliers was digitised by a dual-range 8-bit ADC to increase the dynamic nge, with the conversion ratio chosen according to the type of module, in a fashion nilar to that adopted in the CHARM experiment [8].

The modules to be tested were placed on a support that could move in the o directions perpendicular to the beam line of flight, thus allowing to study possible pendence of the performance on the position of the impact point of the beam on e module. This impact point was defined with an accuracy of $\sim 1 \mathrm{~cm}$ and angular solution of $\sim 1 \mathrm{mrad}$, with the help of the counters $F_{1}$ and $F_{2}$, made out of four smaller intillators of $1 \mathrm{~cm} \times 1 \mathrm{~cm} \times 8 \mathrm{~cm}$ each.

Data were taken at electron momenta varying between 2.5 and $10 \mathrm{GeV} / \mathrm{c}$ and with $0 \mathrm{GeV} / \mathrm{c}$ muons. Different types of modules were tested individually and, to achieve od containment for electromagnetic showers, six modules of the EM and six of HAD1 pes were packed together to form two walls, as shown in the insert of Fig. 7 with a pth of comparable number of radiation lengths $\left(\sim 17 X_{0}\right.$ for EM, $\sim 22 X_{0}$ for HAD1). 


\section{Analysis and results}

\section{Light attenuation lengths and longitudinal uniformity}

A typical response to $100 \mathrm{GeV} / \mathrm{c}$ negative muons incident at the centre of each the three types of modules is shown in Fig. 8. Voltage on the photomultipliers was t so that minimum ionising particles would give a signal whose most probable value, nen digitised, corresponded to a channel between 25 and 50 of the high sensitivity rt of the dual-range ADC. Fig. 9 shows the response of the detectors in the W1, W2 d W3 configurations to electrons of $3 \mathrm{GeV} / \mathrm{c}$ nominal momentum, where losses due to agitudinal leakage are expected to be small.

To determine attenuation lengths and to study the longitudinal uniformity of the ree types of detectors, a horizontal scan over different impact points of the beam was rformed.

For the EM-type modules, the attenuation length was determined using the most obable value of the energy released by $100 \mathrm{GeV} / \mathrm{c}$ muons (peak of Landau distribution) a function of distance from the photomultiplier ${ }^{\dagger}$. The data are shown in Fig. 10a, nere the line represents the result of a fit to a single exponential form of the type $e^{-x / \lambda}$ the points. Averaging over ten modules, a mean value of $462 \mathrm{~cm}$ for $\lambda$ was obtained, th a spread of $53 \mathrm{~cm}$ about this mean value. In Fig. 10b the sum of the pulse heights photomultipliers situated at opposite sides is plotted for various impact points of the am along the module and it exhibits the expected uniform behaviour, namely a signal ich depend only very weakly upon the beam incidence point. A similar behaviour (see g. 10c) is also observed with electrons incident on the W1 configuration (EM-wall). In is case the signals from all the photomultipliers connected to all the modules that ake up the wall are combined. Systematic differences in the response of the detector nen particles are incident at the centre or at the edge of the module are very small. ne dashed line in Fig. 10c represents the signal averaged over the different impact ints. The ratio between the rms spread of the points about this mean value and the erage, gives an estimate of the degree of uniformity of the EM-wall. For electrons of $\mathrm{reV} / \mathrm{c}$ momentum, this ratio was less than $1 \%$.

Fig. 11a shows the results of the analysis of data taken with a $3 \mathrm{GeV} / \mathrm{c}$ electron am hitting the central longitudinal axis of a read-out cell of HAD2 (the part of the lorimeter equipped with scintillator strips), at five different impact points, starting 
ident, in the data taken with filters. Averaging over the five HAD2 modules tested in e X7 beam (corresponding to ten read-out cells), the mean attenuation length with ters is $362 \mathrm{~cm}$ with $50 \mathrm{~cm}$ rms spread and $(251 \pm 30) \mathrm{cm}$ without filters. As it is shown in g. 11b, the sum of left and right pulses shows little dependence on the distance of the rticle impact point from the photomultiplier $(<10 \%)$ while the maximum deviation easured without filters amounts to $\sim 25 \%$.

A systematic study of the effect of filters was made only for the HAD2-type odules. However some measurements were repeated using the HAD1 modules and these nfirmed the conclusions, as it might have been expected since they are equipped th the same type of photomultipliers and the emission spectra of the fibers and the intillator strips are similar.

Fig. 12a and 12b show the response of one HAD1-type module to $3 \mathrm{GeV}$ electrons as function of the distance from the photomultiplier. As expected, they exhibit the same haviour as the other detectors. When averaged over ten modules, for the attenuation igth $\lambda$, a value of $220 \mathrm{~cm}$ with a $20 \mathrm{~cm}$ rms spread about this mean value, is obtained thout filters. An improvement of $\sim 30 \%$ is obtained when filters are used.

Since physical processes are observed by photomultipliers at both ends of the odule, it is possible to estimate the number of photoelectrons produced at the cathode ing the relation

$$
N_{p e}=\frac{2<N>^{2}}{\sigma_{\Delta}^{2}}
$$

nere $\sigma_{\Delta}$ is the standard deviation of the distribution of the difference of left and sht ADC counts and $\langle N\rangle$ is their common average value. This formula is valid the approximation of complete symmetry left-right in light propagation, collection d photocathode efficiencies. It was found that, without filters for HAD1 and HAD2, nen electrons of energy $E$ are incident at the center of the module, the cathode of single photomultiplier would produce $\sim 170 \cdot E, \sim 280 \cdot E$ and $\sim 270 \cdot E$ (with $E$ in $\mathrm{e} V$ ) photoelectrons. The use of filters in HAD1 and HAD2 of course reduces the number photons reaching the photocathode. It was computed that in these conditions the Imber of photoelectrons per $G e V$ is reduced to $\sim 140$ and $\sim 110$ respectively for the ro detectors. The effect of this reduction on the amplitude of the signal was partly mpensated by increasing the operating voltage of the photomultipliers. This does not nstitute a problem however, since the absolute number photoelectrons is adequate and es not deteriorate appreciably the energy resolution (see section 5.2). Furthermore, these conditions the modules are capable of detecting with full efficiency minimum aising particles that cross at the far end (about $3.7 \mathrm{~m}$ for HAD2). 


\begin{tabular}{||l||c|c||c|c||}
\hline EM & & $u$ & $u$ & 0 \\
\hline HAD1 & $0.139 \pm 0.003$ & $0.001 \pm 0.001$ & $0.141 \pm 0.002$ & $0.007 \pm 0.005$ \\
\hline HAD2 & $0.125 \pm 0.005$ & $0.005 \pm 0.002$ & $0.131 \pm 0.003$ & $0.016 \pm 0.003$ \\
\hline
\end{tabular}

ble 2: Values of parameters obtained fitting the resolution with the functions described in the xt.

e neutrino oscillation experiment CHORUS, its geometry is transverse to the direction the incident particles, contrary to the configuration typically envisaged for collider plications. The use of scintillating material (fibers and strips) of excellent quality as paramount to overcome potential difficulties related to this geometry and to the nsiderable size of the modules. Measured attenuation lengths of more than $3 \mathrm{~m}$ and ht collection at both ends ensure small corrections and good uniformity. Results from exposure of the different modules to an electron beam, indicate that the response electromagnetic showers agrees with the expectations, as far as linearity and energy solution are concerned. The response of the calorimeter as a whole to electrons and ons will be studied in a forthcoming test, where the high resolution of the calorimeter hadrons, required by the experiment, will be verified.

\section{Acknowledgments}

The design, construction and installation of this calorimeter in less than one ar would have not been possible without the constant support of the technical staff the collaborating institutions and of CERN. In particular, special thanks are due to to Carassiti for the design and supervision of the mechanical aspects of the whole oject and to G. Basti, G. Passeggio and R. Gorini for their work on the design. The lp of S. Bigoni, G. Bonora, F. Evangelisti, G. Improta, A. Iaciofano, M. Melchiorri, Rocco and S. Sabbioni in the design and construction of the large number of tools cessary to make the assembly easier and for the actual assembly of the modules and the calorimeter itself, is gratefully acknowledged. For the construction of EM modules INFN Naples, we express our appreciation for the skillfull work of M. Borriello, C. aiazzo, D. Cocozza, F. Esposito, C. Farella, A. Improta, V. Marzullo, C. Molino, F. gano and G. Vanzanella. The mechanical workshop and the drawing office of INFN me were essential in the design and construction of the machine for module assembly. lanks are also due to the personnel of Pol. Hi. Tech. at Carsoli for the care during e construction and to F. Pulcinella for his work on the electronics necessary for the adout. The efforts of the personnel from the administrative offices made material 
References

I. D. Acosta et al., Nucl. Instr. and Meth. A294 (1990) 193.

2. D. Acosta et al., Nucl. Instr. and Meth. A308 (1991) 481.

3. N. Armenise et al., CHORUS Collaboration, CERN - SPSC/90-42; M. de Jong et al., CHORUS Collaboration, CERN - PPE/93-134.

4. S. Buontempo et al., Nucl. Phys. (Proc. Suppl.) B32 (1993) 70; S. Buontempo et al., presented at the III International Conference on Calorimetry in High Energy Physics, Chorpus Christi, USA, 1992.

5. J. P. DeWulf et al., CHARM II Collaboration, Nucl. Instr. and Meth. A252 (1986) 443; Nucl. Instr. and Meth. A263 (1988) 109; Nucl. Instr. and Meth. A278 (1989) 670.

3. S. Buontempo et al., presented at the 5th Topical Seminar on Experimental Apparatus for High Energy Physics and Astrophysics S. Miniato, Italy, 1993.

7. M.A. Mazzoni and P. Zucchelli, DAQ System for the calorimeter test, Internal Note (1992) (unpublished).

3. A.N. Diddens et al., Nucl. Instr. and Meth. 178 (1980) 27.

. L. Gatignon, private communication.

10. G. Anzivino, presented at IV International Conference on Calorimetry in High Energy Physics, La Biodola, Isola d'Elba, Italy, 1993.

11. S. Miscetti, for the KLOE Collaboration, presented at IV International Conference on Calorimetry in High Energy Physics, La Biodola, Isola d'Elba, Italy, 1993. 
Isometric view of the calorimeter.

(a) Cross section of EM-type module. The lead sheets are perpendicular to the beam direction, represented by the arrow. (b) Details of grouping of scintillating fibers and optical coupling in EM-type modules. The metal housing for the photomultipliers is also shown.

Grooved lead sheet that constitutes the basic building element of EM and HAD1-type modules.

Details of HAD1-type modules. Shown are cross section, grouping of fibers (hexagonal bundle) and housing for light guide and photomultiplier. Note that the orientation with respect to the beam direction is different from that of EM-type modules. Cross section of HAD2-type module. Visible are also the screws that hold the lead sheets in the modules placed vertically.

Details of the optical coupling for the HAD2-type modules.

Experimental setup used during the test of some of the modules in the X7B beam at CERN.

Response of the three types of modules to muons of $100 \mathrm{GeV} / \mathrm{c}$ momentum. (a) EM; (b) HAD1; (c) HAD2.

Response of the three types of detectors to electrons of $3 \mathrm{GeV} / \mathrm{c}$ nominal momentum. (a) EM; (b) HAD1; (c) HAD2.

Performance of EM-type modules. (a) Response to $100 \mathrm{GeV} / \mathrm{c}$ muons as a function of the distance from the photomultiplier. The dashed line represents the result of a fit of the type $A \cdot e^{-x / \lambda}$; for this particular module an attenuation length of $\lambda=455 \mathrm{~cm}$ was obtained. (b) Sum of the signals from the left and right photomultipliers for different points of incidence of the muon beam along the module. The center of the module is at zero on the horizontal axis. (c) Sum of all signals (left and right photomultipliers) recorded by the six modules in the W1 configuration (see text) for $3 \mathrm{GeV} / \mathrm{c}$ incident electrons, for different impact points of the beam. The center of the module is at zero on the horizontal axis. Note the very small difference between the signal observed with the beam is incident at the center (zero) or at the edge. The dashed line represents the value of the signal when averaged over the different impact points. A measure of the non-uniformity along the module may be given by the dispersion of the individual measurements about the mean value. The rms spread divided by the mean value is 0.008 .

Performance of HAD2-type modules. (a) Signal for $3 \mathrm{GeV} / \mathrm{c}$ electrons as a function of the distance from the photomultiplier with and without the use of yellow filters. The lines are the results of fits of the type $A \cdot e^{-x / \lambda}$. (b) Sum of the signal seen by photomultipliers at both ends as a function of the beam impact point. The lines represent the expected behaviour if the signals collected were attenuated following an exponential law.

As Fig. 11 but for HAD1-type modules. 
are the results of a linear fit performed separately for the three types of modules.

Resolution of the three detectors as a function of the energy of the incident electron. EM:squares; HAD1:triangles; HAD2:circles. The beam impact point is the center of the module. The lines are results of the fit described in the text, performed separately for the three types of modules. 